\title{
Improved accuracy of eddy-current sensor based carrier lifetime measurement using laser excitation
}

\author{
Dávid Krisztián ${ }^{1,4}{ }^{*}$, Ferenc Korsós ${ }^{1}$, Ilias Saegh ${ }^{1}$, Gábor Paráda ${ }^{1}$, Martin Kovács ${ }^{1}$, Zita Verdon ${ }^{1}$, Csaba Jobbágy ${ }^{1}$, \\ Péter Tüttö ${ }^{1}$, Xueqian Dong ${ }^{2}$, Hao Deng ${ }^{3}$, Shasha $W^{2}{ }^{3}$, and Xiaobo Chen ${ }^{3}$ \\ 1 Semilab Co. Ltd. 4/A Prielle K. str., 1117 Budapest, Hungary \\ 2 Semilab Trade Shanghai Co., Ltd., Pudong, Shanghai 201210, P.R. China \\ ${ }^{3}$ LONGi Green Energy Technology Co., Ltd., Xi'an, Shaanxi 710100, P.R. China \\ ${ }^{4}$ Budapest University of Technology and Economics, Institute of Physics, 1111 Budapest, Hungary
}

Received: 14 July 2021 / Received in final form: 8 November 2021 / Accepted: 22 December 2021

\begin{abstract}
In the manufacturing of modern solar cells, the early-phase quality inspection is crucial to detect harmful impurities and to reduce production waste. Thus, the charge-carrier lifetime measurement is beneficial before slicing the silicon ingot into wafers. Optimization of the widely used eddy-current detected photoconductance decay (e-PCD) technique for lifetime measurements is necessary to keep the pace with the new challenges appearing in photovoltaics. We investigated the dependence of the accuracy of the measurable carrier lifetime on the excitation laser parameters in the entire relevant carrier lifetime range. The complex behaviour of surface-recombination phenomena and its time evolution is studied by computer simulation. We optimized the components and parameters of the measurement setup and found that a $1064 \mathrm{~nm}$ laser is rather optimal compared to the $980 \mathrm{~nm}$ laser used previously. Using a longer wavelength laser source with elevated photon flux results in an improved accuracy of carrier lifetime recorded at $\Delta n=10^{15} \mathrm{~cm}^{-3}$ excess carrier concentration. A convincing similarity is found by comparing results from as-sliced and passivated surfaces. The optimized laser e-PCD method was compared to the flash-lamp based PCD method (known as QSSPC). Results agree very well for longer carrier lifetimes for which both systems evaluates the PCD curves in transient mode. However, for lower carrier lifetimes the universal transient mode operation of the e-PCD method is advantageous in terms of consistency.
\end{abstract}

Keywords: Carrier lifetime / silicon ingot / photoconductance decay / eddy-current

\section{Introduction}

The quality inspection of mono-crystalline silicon ingots has increasing importance since this tends to be the dominating material for solar cells [1]. The measured lifetime in this early production phase can indicate the final device performance directly or indirectly [2]. This makes the classification of the different sections of the ingot possible; the high quality parts are cut into wafer, while the lower quality sections can be remelted or classified as scrap.

The photoconductance decay (PCD) method is the standard measurement technique for carrier lifetime characterization of semiconductor materials and devices [3]. This contactless and non-destructive method applies photogeneration to create electron-hole pairs and monitors the corresponding excess conductance decay by an

\footnotetext{
* e-mail: david.krisztian@semilab.hu
}

inductively coupled radio-frequency (RF) coil (e-PCD [4]) or a microwave antenna ( $\mu-\mathrm{PCD}[5])$ after the illumination is terminated.

The carrier lifetime of the Czochralski-grown monocrystalline (Cz-mono) silicon varies within a very broad range in today's photovoltaic industry. In the modern solar cell structures (e.g. heterojunction technology) high carrier lifetime ( $>1 \mathrm{~ms}$ ) is required, while for the manufacturing of the dominating PERC (Passivated Emitter Rear Cell) solar cells low resistivity Ga doped wafers are also commonly used featuring much lower carrier lifetimes $(10-100 \mu \mathrm{s})$. Thus, the accurate and comparable characterization in the low and high carrier lifetime ranges is equally important to properly classify the ingots.

While bulk lifetime $\left(\tau_{\mathrm{b}}\right)$ is the primary parameter indicating the quality, the identification or classification of given defects can be feasible by evaluating $\tau_{\mathrm{b}}$ values as a function of excess carrier density, i.e. the injection level $(\Delta n)$ [6-8]. The aim of our RF e-PCD tool development is to realize such a measurement routine at ingot level without 
Table 1. Technical parameters of the earlier (WT-1200I) and the improved (WT-1200IL) measurement tools.

\begin{tabular}{lll}
\hline & WT-1200I & WT-1200IL \\
\hline Wavelength $(\lambda)$ & $980 \mathrm{~nm}$ & $1064 \mathrm{~nm}$ \\
Penetration depth & $0.1 \mathrm{~mm}$ & $0.85 \mathrm{~mm}$ \\
Photon flux $(\Phi)$ & $3 \times 10^{18} \mathrm{~cm}^{-2} \mathrm{~s}^{-1}$ & $2 \times 10^{19} \mathrm{~cm}^{-2} \mathrm{~s}^{-1}$ \\
Coil diameter & $1.8 \mathrm{~cm}$ & $1.3 \mathrm{~cm}^{-}$ \\
\hline
\end{tabular}

any sample preparation. Now, we are focusing on the achievable accuracy of the measurement by optimizing the illumination using very simple evaluation without complicated corrections. The accuracy of the carrier lifetime value at $10^{15} \mathrm{~cm}^{-3}$ injection level is reported as the standard parameter widely used in the photovoltaic industry.

The measurement of thick samples is both an opportunity and a challenge. An opportunity to obtain bulk lifetime values without any surface treatment and a challenge to suppress the impact of the recombination at the unpassivated front surface. This requires a sufficiently deep photogeneration and the appropriate detection of charge carriers which are realized in our commercial product (Semilab WT-1200I/IL) by using an infrared laser and an inductively coupled radio-frequency coil [9]. The alternative, widespread technique employs flash lamp for photogeneration (QSSPC) with a characteristic decay time of several tens of microseconds or even longer. Pure transient decay mode evaluation is feasible only if the lifetime is significantly longer than the decay of the illumination. For lower carrier lifetime samples, the so-called quasi-steady-state (QSS) mode evaluation needs to be used.

In the evaluation of the PCD measurement from thick samples, the main challenges are the continuously changing rate of the surface recombination and the proper calculation of $\Delta n$. The accuracy of the QSSPC method [10] has been investigated in several studies for both its transient and QSS mode. Swirhun et al. showed first how the excess carrier profile evolves during the transient, and how it affects the measured effective lifetime [11]. Goodarzi et al. later showed that using various long-pass optical filters reduces the impact of the unpassivated surface for both transient [12] and QSS mode [13]. Despite of the thorough optimization, their experimental results showed that the correlation between the different methods in their common range is inappropriate [14], a $40-70 \%$ difference is observable on different segments of the same ingot [11]. It clearly implies that a unified method over the whole relevant lifetime range is very beneficial which is realized in our approach using a fast switching laser for the PCD method.

To make better control over the challenges, we optimized the laser excitation parameters. We investigated the effect of surface recombination at different wavelengths for a wide lifetime range. A 1-D simulation software has been developed to study the change of the impact of the surface recombination during the transient decay. After the primary results, the originally used $980 \mathrm{~nm}$ wavelength has been compared to a rather optimal $1064 \mathrm{~nm}$ laser (emitting elevated photon flux) by simulation and measurements as well. We implemented a novel calculation of injection levels based on the monochromatic nature of the laser excitation. Finally, the reliability of this approach and the influence of surface quality is investigated experimentally by comparing e-PCD results between Cz-mono silicon slabs with as-sliced and chemically passivated surface, and by correlating carrier lifetime values measured by the laser e-PCD and the QSSPC systems.

\section{Laser optimization and simulation}

\subsection{Measurement setup}

Semilab WT-1200I has been developed to perform e-PCD measurement originally for long carrier lifetime ingots [9] using an infrared laser and an RF-coil. It has been designed to be a hand-held tool but the e-PCD sensor can also be integrated into scanning platforms as well. As the importance of the lower carrier lifetime $\mathrm{Si}$ material emerged recently, the accuracy of the e-PCD configuration in this range needed to be revised and further optimized. In this work, we present important steps of this optimization and compare the original tool with the upgraded version called WT-1200IL which has been recently commercialized.

In the original WT-1200I a $980 \mathrm{~nm}$ laser source illuminates the ingots at a $2.2 \mathrm{~cm}$ diameter spot with an average photon flux of $3 \times 10^{18} \mathrm{~cm}^{-2} \mathrm{~s}^{-1}$. The full width at half maximum at the Gaussian-like light distribution coincides the size of opening for the light (referred as spot size). In the upgraded tool the laser has been replaced by an $1064 \mathrm{~nm}$ one which features eight times greater penetration depth (0.85 mm compared to $0.1 \mathrm{~mm}$ ) into silicon. Furthermore, the photon flux has also been increased to $2 \times 10^{19} \mathrm{~cm}^{-2} \mathrm{~s}^{-1}$, the largest value allowed by the $3 \mathrm{~B}$ laser class using the optical design of WT-1200IL system. The technical parameters of the measurement tools are summarized in Table 1.

After the illumination terminates, the conductivity change is monitored by an inductively coupled RF-coil. The coil diameter was reduced compared to the original setup $(1.3 \mathrm{~cm}$ in the present version), being smaller than the illuminated area, but larger than typical diffusion lengths in silicon (Fig. 1a). Using these optimized dimensions we intended to sense the excess carriers in the relatively homogeneous part of the light spot and to minimize the impact of lateral diffusion but still operating the e-PCD systems with sufficiently high photon flux density. 
a)

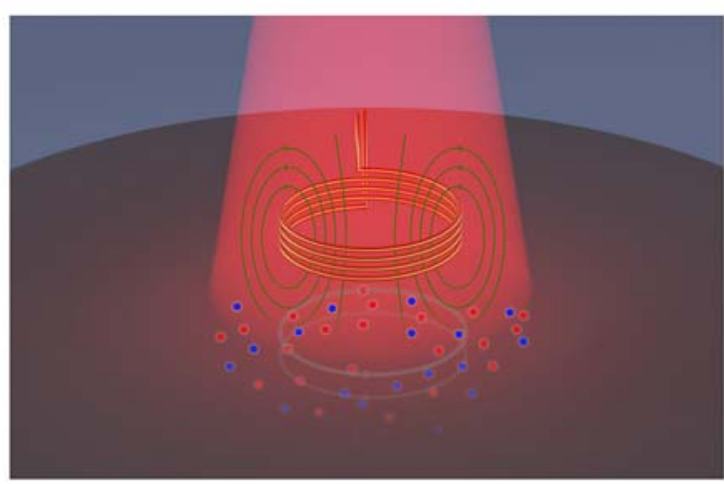

b)

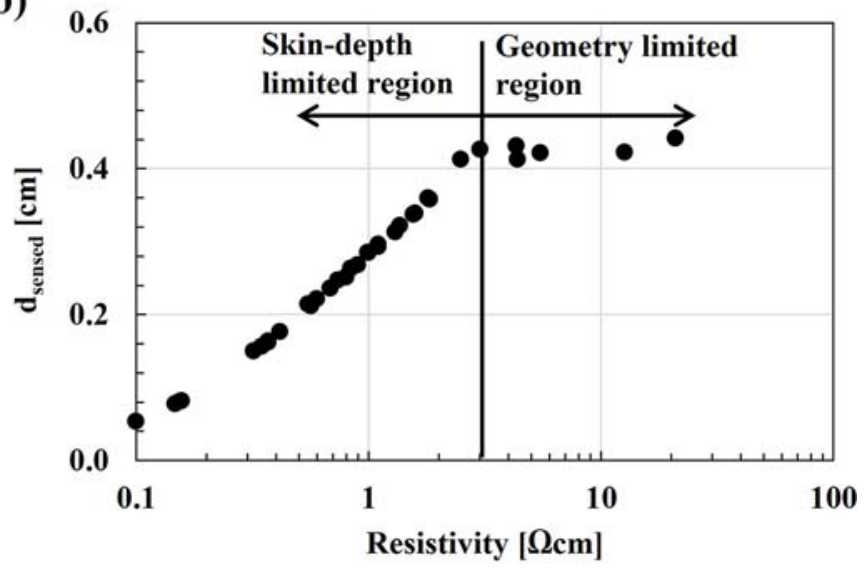

Fig. 1. (a) Schematic illustration of the laser e-PCD measurement method. (b) Effective depth of sensitivity of the RF-coil as measured on thick samples.

To calibrate the depth sensitivity of the coil, we measured the sheet resistance of a set of thin wafers $(\sim 300 \mu \mathrm{m})$ with the e-PCD tool and by four-point-probe technique. We obtained a linear relationship on the relevant sheet conductance range. However, in the case of thick samples such as ingots or slabs (thick slices $>1 \mathrm{~cm}$ thickness) the sensitivity of the e-PCD measurement is limited by the penetration depth of the radio-frequency wave. We determined an effective depth of sensitivity as [11]:

$$
d_{\text {sensed }}=\frac{\rho_{4 \mathrm{pp}}}{\rho_{\square, \text { sensed }}},
$$

where the resistivity, $\rho_{4 \mathrm{pp}}$ is measured by four-point-probe technique, and $\rho_{\square}$,sensed is the sensed sheet resistance calculated using the wafer calibration, both recorded on the same thick samples (several cm thickness). The effective depth of sensitivity is depicted on Figure 1b. in the function of resistivity. In the low resistivity range the effective depth increases as the skin depth increases. Meanwhile over $3 \Omega \mathrm{cm}$, the effective depth is constant, since the geometry of the coil becomes the limiting factor.

The continuous wave lasers are electrically chopped to realize fast switching which is required to perform transient measurement in the $\mu$ s lifetime range as well. The lower limit of the detectable characteristic decay time is determined by the switching time of the laser and the response time of the RF-circuit. $800 \mathrm{~ns} \mathrm{PCD}$ decay time was the shortest we could reliably record using this hardware from a highly contaminated silicon sample. Therefore, samples exceeding $2-3 \mu$ s carrier lifetimes can be reliably characterized using transient mode evaluation. The electric chopping of the laser also allows to vary the pulse length which enables to achieve steady-state photogeneration condition independently of the bulk lifetime of the sample. The measured transients curves are averaged to decrease the noise, but the total time required for the measurement and evaluation is less than $5 \mathrm{~s}$.
The schematic illustration of the e-PCD method is shown in Figure 1a.

\subsection{Charge-carrier profile simulation}

Two main goals have been considered to choose the most appropriate laser wavelength and to determine the accuracy of the measurements: to reduce the influence of the surface recombination and to reach as high chargecarrier density as possible. The depth profile along the $x$ axis ( $x=0$ means the front surface) of the carrier distribution $(\Delta n(x . t))$ needs to be determined first to obtain these quantities [15]. In the most general case, the continuity equation of the excess carriers can be written in the following form [16]:

$$
\begin{aligned}
\frac{\partial \Delta n(x, t)}{\partial t}= & G(x, t)-R(x, t)+D_{\mathrm{amb}}\left(N_{\mathrm{dop}}, \Delta n\right) \\
& \times \frac{\partial^{2} \Delta n(x, t)}{\partial x^{2}}
\end{aligned}
$$

where $G(x, t), R(x, t)$ and $D_{\text {amb }}$ are the generation rate, recombination rate, and ambipolar diffusion coefficient [16], respectively. Drift currents are not included in this model. In the very close proximity of the front surface $(<1 \mu \mathrm{m})$ the possible impact of external electric field is incorporated in the surface recombination velocity (see later). In deeper regions, an internal electric field is induced as a result of faster diffusion of excess holes. Drift currents originating from this effect virtually decelerate the diffusion of electrons and accelerate the diffusion of holes. Therefore can be handled as diffusion with a modified so-called ambipolar diffusion coefficient. In moderately doped silicon the band-band transition dominates the light absorption below $1100 \mathrm{~nm}$ wavelength [17] which means that the electron-hole pair photogeneration rate can be expressed with the absorption coefficient $\alpha$ :

$$
G(x)=(1-r) \Phi \alpha \times \exp (-\alpha x),
$$



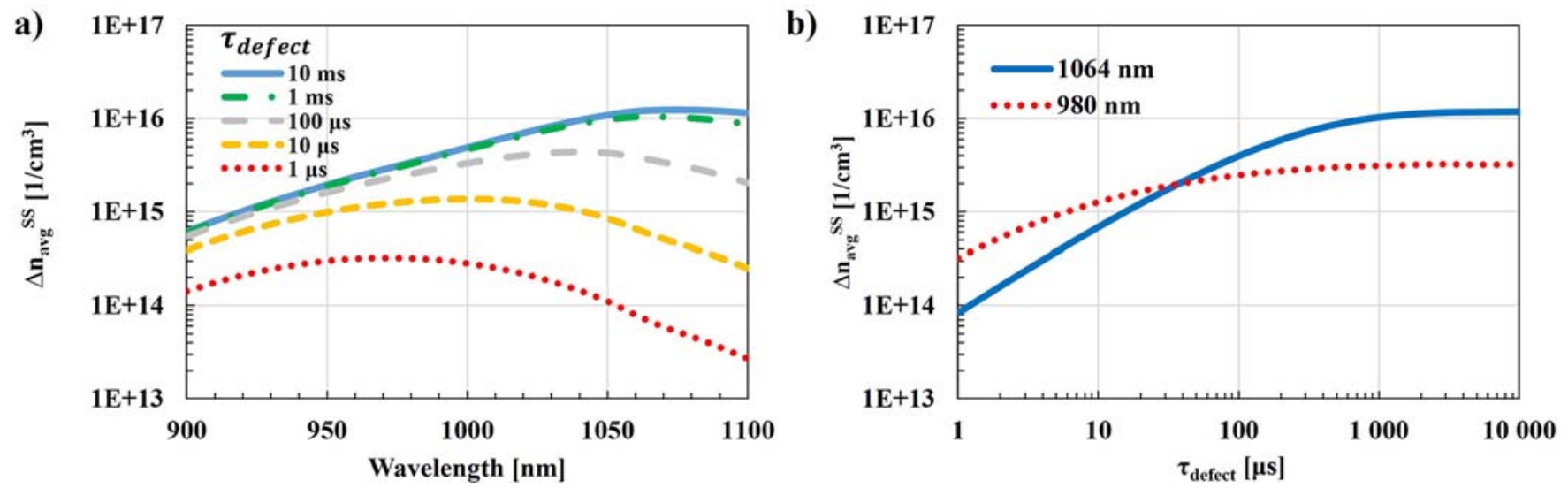

Fig. 2. (a) Steady-state injection levels, $\Delta n_{\mathrm{avg}}^{\mathrm{SS}}$, versus excitation wavelength for samples with different defect related bulk lifetimes. (b) Considering other factors as well, $1064 \mathrm{~nm}$ is proposed for detailed comparison with the earlier standard $980 \mathrm{~nm}$. Parameters: $S_{\text {front }}=10^{6} \mathrm{~cm} / \mathrm{s}, \Phi=2 \times 10^{19} \mathrm{~cm}^{-2} \mathrm{~s}^{-1}$, p-type doping $N_{\text {dop }}=10^{16} \mathrm{~cm}^{-3}$.

where $\Phi$ is the photon flux density and $r$ is the reflected portion of the incident laser power. The recombination rate in the bulk can be expressed as:

$$
R(x, t)=\frac{\Delta n(x, t)}{\tau_{b}(\Delta n)} .
$$

The boundary conditions can be specified by introducing the surface recombination velocity at the front surface $\left(S_{\text {front }}\right)[3]$ :

$$
\begin{aligned}
\Delta n(x & =0, t) \times S_{\text {front }} \\
& =D_{\mathrm{amb}}\left(N_{\mathrm{dop}}, \Delta n\right) \times\left.\frac{\partial \Delta n(x, t)}{\partial x}\right|_{x=0} .
\end{aligned}
$$

Equation (2) is a partial differential equation which cannot be solved analytically for a general case. Therefore, we resort to a 1-D computer simulation to determine the excess carrier profile during the transient decay. In this simulation, the bulk recombination rate consists of the intrinsic recombination rate (using the model proposed by Richter et al. [18]) and a defect-related recombination rate. To simplify the discussion, we consider the injection level dependence of the intrinsic recombination, while defect related recombination mechanisms are treated as independent of injection level. Thus, we focus on the influence of surface recombination but avoiding to calculate unrealistic injection level and carrier lifetime.

$$
\frac{1}{\tau_{b}\left(N_{\mathrm{dop}}, \Delta n\right)}=\frac{1}{\tau_{\mathrm{intr}}\left(N_{\mathrm{dop}}, \Delta n\right)}+\frac{1}{\tau_{\text {defect }}} .
$$

The ambipolar diffusion coefficient depends on injection level. It is also taken into account in the simulations considering the injection level dependence of the electron and hole mobility as well using using the model of Dannhauser [19] and Krausse [20].

The solution of the Equation (2) differential equation is a strongly inhomogeneous excess carrier depth profile evolving in time during the measurement. However, a practical parameter, the mean excess carrier density $\left(\Delta n_{\text {avg }}\right)$ can be defined using $\Delta n(x)$ as a weight function [10]:

$$
\Delta n_{\text {avg }}(t)=\frac{\int_{0}^{W}(\Delta n(x, t))^{2} d x}{\int_{0}^{W} \Delta n(x, t) d x} .
$$

This way a spatially averaged injection level can be determined, from which a spatially averaged lifetime is calculated as the result of the evolution of measured transients. Such approximation is necessary to define the injection level in the case of thick samples for which the carrier lifetime is reported, although these value may differ from values taken from thin wafer with excellent surface passivation.

Reaching $10^{15} \mathrm{~cm}^{-3}$ excess carrier density is essential since carrier lifetime is usually reported on this density in the standard practice. Increasing the photon flux obviously enhances the steady state injection level, $\Delta n_{\mathrm{avg}}^{\mathrm{SS}}$, but it is limited by radiation safety regulations in commercial systems. For all simulations, we used the maximum photon flux, allowed by the 3B laser class $\left(\Phi=2 \times 10^{19} \mathrm{~cm}^{-2} \mathrm{~s}^{-1}\right.$ in the optical design of WT-1200IL system). Besides the photon flux, the wavelength of the excitation laser needs to be optimized since the absorption coefficient strongly depends on it [17]. The steady-state carrier injection level in the wavelength range of 900 to $1100 \mathrm{~nm}$ is depicted in Figure 2 for different $\tau_{\text {defect }}$ values. The penetration depth in silicon is a strongly increasing function of the wavelength in this range, and this affects significantly for the steady state injection level. In the short wavelength range, the excess carriers are generated near to the surface which indicates that the surface recombination limits the $\Delta n_{\mathrm{avg}}^{\mathrm{SS}}$. On the other hand, in the long wavelength and so penetration depth range the excess carriers are generated in a deep volume which also decreases the average injection level. Increasing $\tau_{\text {defect }}$ and so the corresponding diffusion length, the maximal value of $\Delta n_{\mathrm{avg}}^{\mathrm{SS}}$ shifts towards longer wavelengths.

Besides the injection level, the surface recombination must be considered, since it can be significant in the first part of the decay which causes strongly varying carrier 
lifetimes reported from the initial part of the decay curve. As it is shown later, the deeper penetration introduced by longer wavelengths reduces the impact of the surface recombination in this initial decay period.

Considering all these factors, the commercially available $1064 \mathrm{~nm}$ wavelength laser has been selected for the upgraded tool, and it is compared to the originally used $980 \mathrm{~nm}$ one. The comparison of maximum injection levels depicted in Figure $2 \mathrm{~b}$ for the entire $\tau_{\text {defect }}$ range of interest. One can observe in Figure $2 b$ that the steady-state excess carrier density has a crossover point around $\tau_{\text {defect }} \approx 30 \mu \mathrm{s}$. Above this lifetime the new laser appears to be beneficial from this point of view. $10^{15} \mathrm{~cm}^{-3}$ excess carrier density is reached above $\tau_{\text {defect }} \approx 15 \mu$ s which is the lower limit for the mono-Si to manufacture solar cells with acceptable efficiency.

\subsection{Simulation of the laser e-PCD measurement}

To simulate the measured photoconductance signal during the measurement $\Delta V(t)$, which is treated proportional to the sheet density of the "sensed" excess carriers $\Delta N_{\text {sensed }}(t)$, we assumed an exponentially decaying depth sensitivity using $d_{\text {sensed }}(\rho)$ (see Eq. (3)) in the exponent:

$$
\Delta V(t) \approx \Delta N_{\text {sensed }}(t)=\int_{0}^{W} \Delta n(x, t) \times \exp \left(-\frac{x}{d_{\text {sensed }}(\rho)}\right) d x,
$$

where $W$ is the width of the sample. In the general case, this simple approximation for the depth sensitivity and the linearity between the $\Delta V(t)$ and $\Delta N_{\text {sensed }}(t)$ is not valid. Due to the inhomogeneous $\Delta n(x)$ depth profile, $d_{\text {sensed }}$ gets dependent on the actual carrier concentration at a given depth, so $\Delta d_{\text {sensed }}(\rho, n(x))$ get varying in depth. In addition, the injection level dependence of the electron and hole mobility should be also considered when converting $\Delta N_{\text {sensed }}(t)$ to $\Delta V(t)$. This is a quite complex topic which is going to be addressed in a separate work later. However, since the results reported at $\Delta n=10^{15} \mathrm{~cm}^{-3}$ are in the focus of this study, it is sufficient to use $d_{\text {sensed }}(\rho)$ which is constant in depth (but varies with resistivity) in the simulations, since $\Delta n<N_{\text {dop }}$.

In the practice and in the simulations, the duration of the laser pulse is set long enough to achieve steady state conditions. Therefore, the steady state injection level, before the termination of the laser pulse, can be calculated if the bulk carrier lifetime is known. The evaluation of the recorded PCD transients starts with the estimation of $\Delta n_{\text {avg }}^{\mathrm{SS}}$ based on the results shown in Figure $2 \mathrm{~b}$ and using an estimation of the bulk lifetime as input parameter applying the e-PCD curve fitting described in [9]. During the transient decay the average injection level $\Delta n_{\text {avg,meas }}(t)$ is considered to be proportional to the signal:

$$
\Delta n_{\text {avg,meas }}(t)=\frac{\Delta n_{\mathrm{avg}, \text { meas }}^{\mathrm{SS}}}{\Delta V_{\max }} \times \Delta V(t) .
$$

Then, the measured lifetime $\tau_{\text {meas }}$ is calculated from the standard dynamic definition of carrier lifetime from the recorded (and simulated) transients:

$$
\tau_{\text {meas }}(t)=\frac{n_{\text {avg,meas }}(t)}{-\frac{\partial n_{\text {avg,meas }}(t)}{\partial t}} .
$$

The simulated e-PCD transients, carrier depth profiles and evaluated carrier lifetimes and injection levels are summarized in Figure 3 corresponding to a p-type, $N_{\text {dop }}=1 \times 10^{16} \mathrm{~cm}^{-3}$ gallium doped Si ingot with unpassivated surface featuring $\tau_{\text {defect }}=300 \mu$ s defect related bulk lifetime. Simulations for both lasers were accomplished supposing the same $\Phi=2 \times 10^{19} \mathrm{~cm}^{-2} \mathrm{~s}^{-1}$ photon flux for the sake of comparability.

The excess carrier profiles are depicted on Figure $3 \mathrm{a}$ in steady state condition and during the transient decay. The deeper photogeneration of the $1064 \mathrm{~nm}$ laser results in a higher excess carrier density in steady state. Furthermore, the shape of the carrier profile is deeper which is moving even deeper in time during the measurement. The deeper profile means smaller relative rate of the surface recombination, and so longer decay times. This manifests in the simulated PCD transients as well (Fig. 3b), resulting in larger amplitude and longer decay characteristics corresponding to the $1064 \mathrm{~nm}$ laser.

$\Delta n_{\mathrm{avg}}^{\mathrm{SS}}(t)$ and $\tau_{\text {meas }}(t)$ during the measurements are depicted in Figures $3 \mathrm{c}$ and $3 \mathrm{~d}$. The larger initial injection level introduced by the $1064 \mathrm{~nm}$ laser prolongs the time within the injection level decrease to $10^{15} \mathrm{~cm}^{-3}$ (Fig. 3c). The longer time enables deeper depth profile and so reduces influence of the surface recombination. This is clearly visible in Figure 3d as the measured lifetimes get longer with the elapsed time due to the deepening carrier profile.

This is even more evident on Figure 3e, where the lifetime is depicted in the function of the average injection level.

Due to the Auger recombination, the bulk lifetime itself (plotted with black dashed line) is a strong function of the injection level. However, this is the real physical parameter could be measurable by an ideal method at a given injection level. Therefore, we define the theoretical "accuracy" the e-PCD measurement (using the pure, simple transient evaluation) by comparing $\tau_{\text {meas }}$ to the theoretical $\tau_{\mathrm{b}}$ corresponding to a given $\tau_{\text {defect }}$. At $\Delta n=10^{15} \mathrm{~cm}^{-3}$ the measured lifetime is around $75 \%$ of the bulk lifetime with the $1064 \mathrm{~nm}$ laser, while only $50 \%$ with the $980 \mathrm{~nm}$ one around this $\tau_{\mathrm{b}}=300 \mu \mathrm{s}$ range.

A very simple approach was used to calculate $\Delta n_{\text {avg,meas }}(t)$ supposing proportionality between the signal and the average injection level after the steady state conditions. We examined the validity of this approximation by comparing the $\Delta n_{\mathrm{avg}, \text { meas }}(t)$ to the real value of $\Delta n_{\mathrm{avg}}(t)$ calculated from the profile (see Eq. (7)). For the $1064 \mathrm{~nm}$ laser the deviation is very low, below $10 \%$ (since even in steady state conditions the carrier profile is deep enough), while $\Delta n_{\text {avg,meas }}$ can differ very significantly from the real value. 
a)

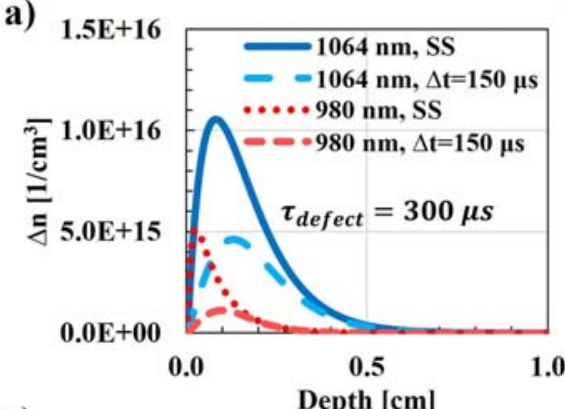

c)

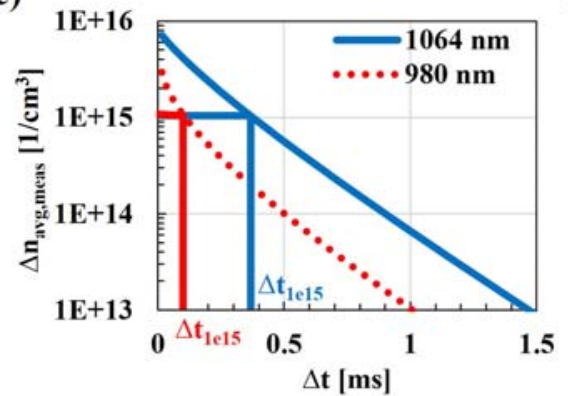

e)

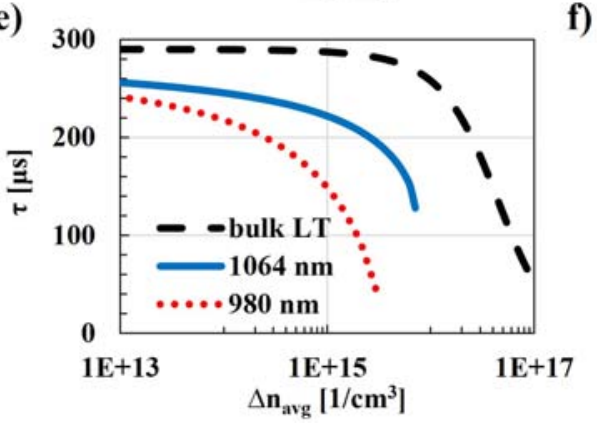

b)

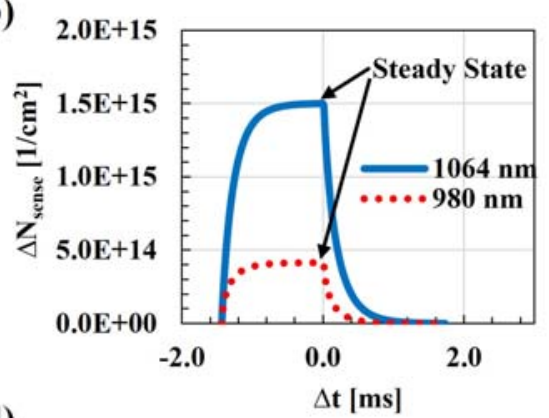

d)

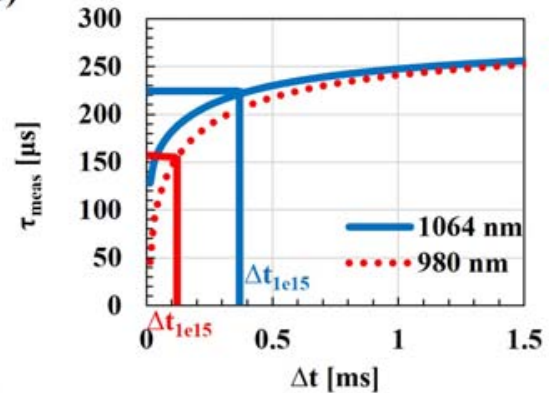

f)

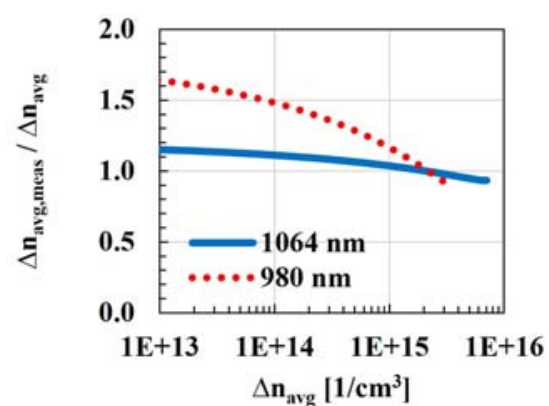

Fig. 3. Simulation of the e-PCD measurement of a gallium doped ingot with $N_{\text {dop }}=10^{16} \mathrm{~cm}^{-3}$ doping density and $\tau_{\text {defect }}=300 \mu \mathrm{s}$ lifetime. (a) Excess carrier depth profiles in steady-state conditions and $\Delta t=150 \mu \mathrm{s}$ time after the illumination is terminated. The depth of the maximal injection level moves towards the bulk as the near-surface carriers recombine rapidly at the surface. (b) Decay of the detected signal. Note that the steady-state condition can be always achieved by varying the pulse length. The excess carrier density (c) and the calculated lifetime (d) in the function of time elapsed. (e) The measured lifetime is more accurate with the $1064 \mathrm{~nm}$ laser.

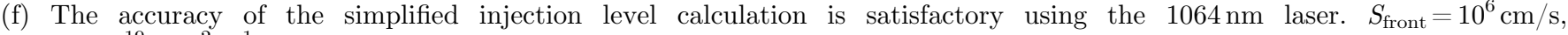
$\Phi=2 \times 10^{19} \mathrm{~cm}^{-2} \mathrm{~s}^{-1}$.

In conclusion, the simulation of the given sample, which represents realistic material properties in production, indicates the crucial importance of laser power and injection level. Using longer wavelength and large enough laser power the impact of the surface recombination is significantly reduced when calculating the carrier lifetime at $\Delta n_{\text {avg,meas }}=10^{15} \mathrm{~cm}^{-3}$, since both the initial carrier

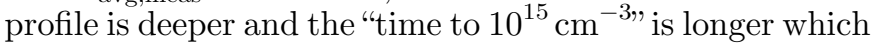
further deepens the profile. This makes possible to record carrier lifetimes and injection levels very close to the nominal value, even using very simple evaluation routine.

\subsection{Accuracy over the entire carrier lifetime range}

We repeated the simulation for different $\tau_{\text {defect }}$ values over a wide range. We focus on $10^{15} \mathrm{~cm}^{-3}$ excess carrier density, so the accuracy of lifetime and injection level is also compared at this condition. The ratio of $\tau_{\text {meas }}\left(10^{15} \mathrm{~cm}^{-3}\right)$ and $\tau_{\mathrm{b}}\left(10^{15} \mathrm{~cm}^{-3}\right)$ is plotted in Figure 4a. One can observe that, similar to the specific case discussed in details $\left(\tau_{\text {defect }}=300 \mu \mathrm{s}\right), \tau_{\text {meas }}$ is much closer to the bulk lifetime over the whole range. In general, the larger the bulk lifetime and the corresponding diffusion length, the greater the portion of the charge carriers that can recombine at the surface decreasing the measured lifetime in the long lifetime range. However, using the $1064 \mathrm{~nm}$ laser the measured lifetime always exceeds the $60 \%$ of the bulk lifetime in case of any realistic samples.

The injection level accuracy at $\Delta n_{\text {avg }}=10^{15} \mathrm{~cm}^{-3}$ in the function of the $\tau_{\text {defect }}$ is demonstrated in Figure $4 \mathrm{~b}$. It clearly indicates the validity of the very simple calculation practically for both lasers. 

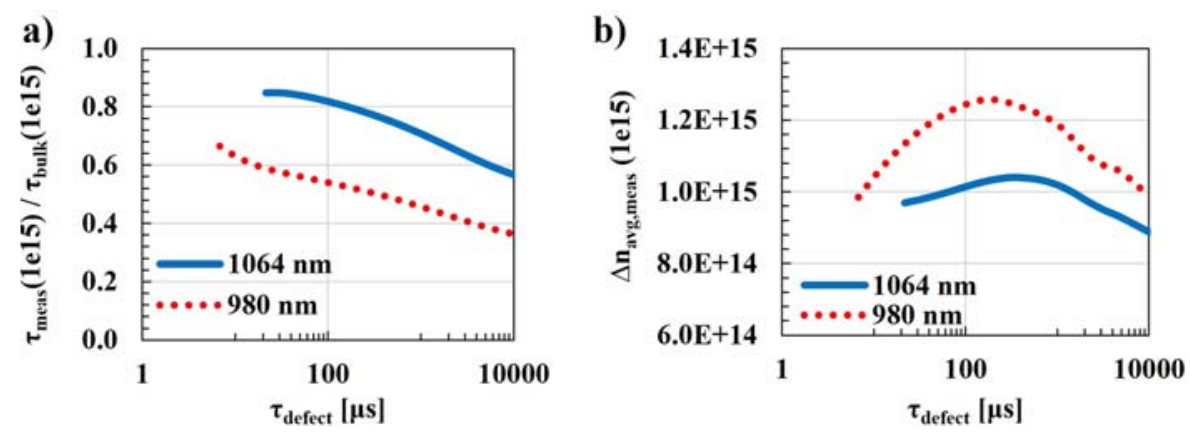

Fig. 4. The accuracy of the measured lifetime (a) and injection level (b) at $10^{15} \mathrm{~cm}^{-3}$ excess carrier density is much better using the $1064 \mathrm{~nm}$ laser. $N_{\text {dop }}=10^{16} \mathrm{~cm}^{-3}, S_{\text {front }}=10^{6} \mathrm{~cm} / \mathrm{s}, \Phi=2 \times 10^{19} \mathrm{~cm}^{-2} \mathrm{~s}^{-1}$.

Finally, we examined the dependence of the measured lifetime on surface recombination velocity, $S_{\text {front }}$ (Fig. 5). At ingot level this value typically lies between $10^{4} \mathrm{~cm} / \mathrm{s}$ and $10^{6} \mathrm{~cm} / \mathrm{s}$ depending on the surface treatments (as-cut, etched, or anything not intentionally passivated). In this range, the simulated measurement outputs does not exhibit significant variation using the $1064 \mathrm{~nm}$ laser. Therefore, the e-PCD measurement is not sensitive for the actual value of the surface recombination velocity, if the surface is not intentionally passivated.

\section{Experiments and results}

\subsection{Validation of the laser upgrade}

We investigated the benefits arising from laser optimization by performing e-PCD measurements on ingots covering a wide lifetime range. A Semilab WT-1200I/IL system was used for the measurements using the original $(980 \mathrm{~nm})$ and the optimized $(1064 \mathrm{~nm})$ laser sources. Using the optimized setup the samples are both measured with $3 \times 10^{18} \mathrm{~cm}^{-2} \mathrm{~s}^{-1}$ (low power) and $2 \times 10^{19} \mathrm{~cm}^{-2} \mathrm{~s}^{-1}$ (high power) photon flux to separate the impact of the different wavelength and light power.

To demonstrate the importance of both the power and wavelength optimization, the measured $\tau_{\text {meas }}$ values are plotted in the function of $\Delta t$ for three selected samples in Figure 6 . First, we examined a high purity n-type sample usually featuring negligible injection level dependence (ILD) of the lifetime at low injection levels (Fig. 6a). This injection level independent behaviour is more typical in case of n-type silicon, since the capture cross coefficients of electrons is usually much higher than that of holes in case of metallic contaminants, such as interstitial iron [21].

There is no significant difference between the results measured at different laser powers and wavelengths. However, at $\tau_{\text {meas }}(\Delta t=0 \mu \mathrm{s})$, the carrier lifetime right after the illumination is terminated is lower measured using the $980 \mathrm{~nm}$ laser as compared to $1064 \mathrm{~nm}$ at low power, due to the larger impact of surface recombination. Furthermore, this initial lifetimes is shorter operating the $1064 \mathrm{~nm}$ laser at high power compared to low power, since Auger recombination is reduces the bulk lifetime at the higher

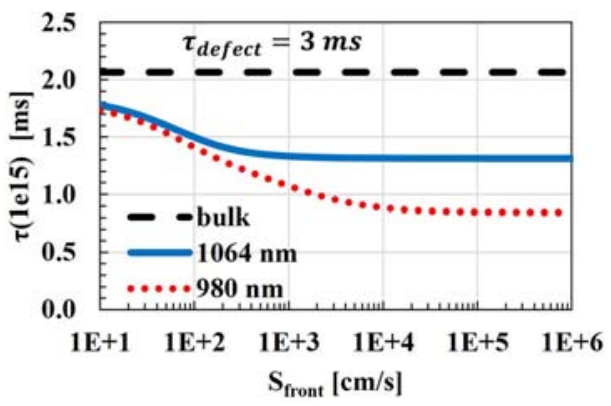

Fig. 5. Measured effective lifetime at $\Delta n_{\text {avg }}=10^{15} \mathrm{~cm}^{-3}$ is independent of the front surface recombination velocity, $S_{\text {front }}$, in its typical range. $N_{\text {dop }}=10^{16} \mathrm{~cm}^{-3}, \tau_{\text {defect }}=3 \mathrm{~ms}, \Phi=2 \times 10^{19} \mathrm{~cm}^{-2} \mathrm{~s}^{-1}$.

injection level. Overall, due to the negligible ILD, the maximal measurable lifetimes are similar, independent of the laser power properties. Therefore, the purity of such ingots and slabs can be reliably qualified even using the original WT-1200I setup [9].

Results from other bulk samples with moderate and strong ILD (Figs. 6b and 6c respectively) clearly demonstrate the importance of laser properties regarding measurement quality. Both samples show an increase in the effective lifetime at the first part of the decay similarly to Figure 6a, but after a certain point $\tau_{\text {meas }}$ becomes lower due to the ILD. The longer wavelength and higher laser power results in an increased maximal lifetime due to the higher injection level achieved. The stronger the ILD, the larger the difference between the results recorded by the $980 \mathrm{~nm}$ and $1064 \mathrm{~nm}$ lasers. It indicates the real importance of using the optimized laser excitation for industrial applications, where samples with strong ILD frequently occur.

\subsection{Correlation tests}

After receiving experimental evidence for the advantage of changing the wavelength to $1064 \mathrm{~nm}$, we applied the injection level calculation method presented in Section 2.3 to study the measured effective lifetimes at certain injection levels. 

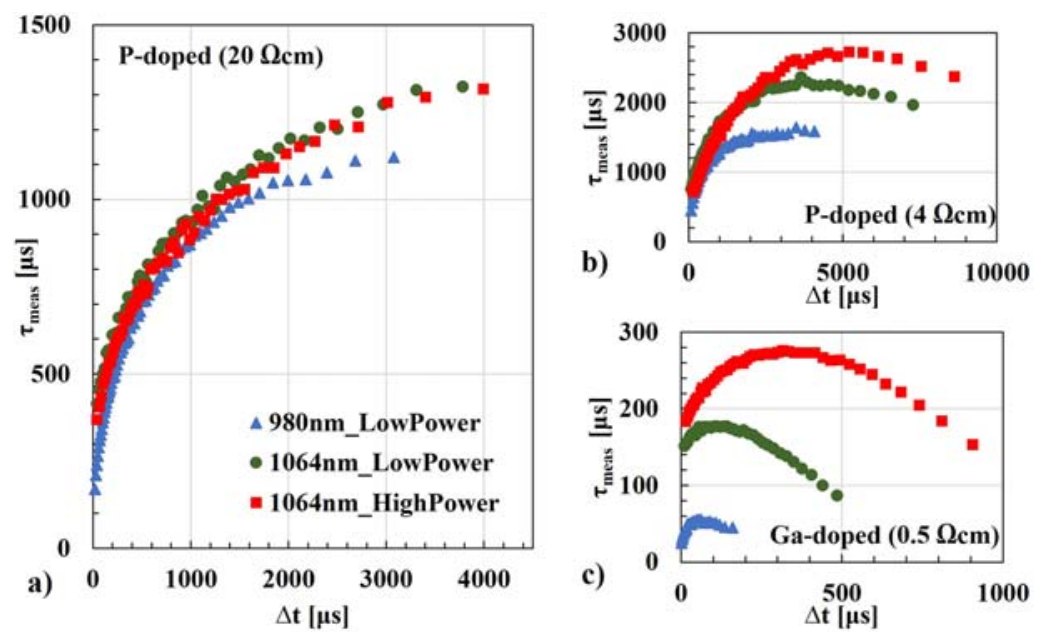

Fig. 6. Measured $\tau_{\text {meas }}$ curves during the PCD decay. (a) The high quality n-type slab featuring negligible injection level dependence (ILD) shows a similarly increasing $\tau_{\text {eff }}(t)$ regardless of laser wavelength and power. In the case of stronger ILD (b and c) longer wavelength and elevated laser power makes possible to determine the lifetime closer to the saturation value of $\tau_{\mathrm{b}}$. (Low power: $3 \times 10^{18} \mathrm{~cm}^{-2} \mathrm{~s}^{-1}$ used in WT-1200I, High power: $2 \times 10^{19} \mathrm{~cm}^{-2} \mathrm{~s}^{-1}$ is used in WT-1200. IL).
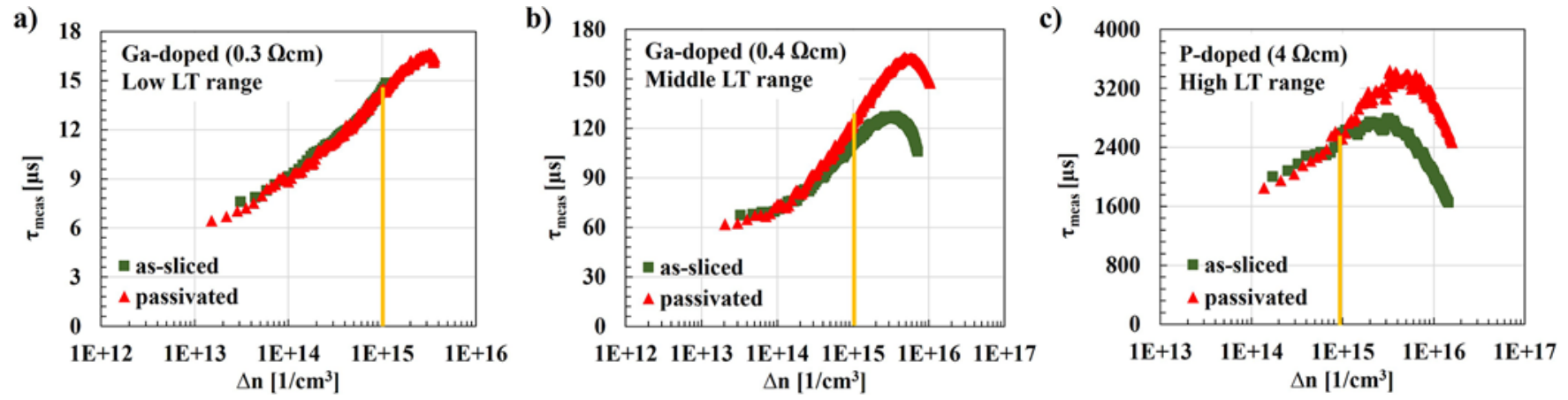

Fig. 7. Effective lifetime measured on as-sliced surface approaches the lifetime from surface passivated Cz-mono silicon slabs. The higher the lifetime, the longer the time needed to approach. At standard excess carrier density $\Delta n=10^{15} \mathrm{~cm}^{-3}$ (marked with yellow lines) and below the difference is under $20 \%$ in both low- (a), middle- (b) and high (c) lifetime cases. During the measurement the excess carrier density decreases, so the time passes from right to left on the x-axis.

The influence of the surface recombination at different injection levels was also examined experimentally. For this purpose, $2 \mathrm{~cm}$ thick $\mathrm{Cz}$-mono silicon slabs were measured using the optimized laser e-PCD tool both with as-sliced and chemically passivated surface preparations. Nine Ga-doped and two P-doped slabs were selected which cover the entire industrially relevant lifetime range. For thorough chemical passivation [5], the chemical damage etching $(30 \mu \mathrm{m}$ thick Si layer from the top surface was removed) was followed by the application of an iodine-ethanol solution. For chemically passivated samples, the front surface recombination velocity $\left(S_{\text {front }}\right)$ lies between $50 \mathrm{~cm} / \mathrm{s}$ and $200 \mathrm{~cm} / \mathrm{s}$ [5] and was estimated to be $100 \mathrm{~cm} / \mathrm{s}$ for the 1-D simulation which was used to calculate $\Delta n_{\mathrm{avg}}^{\mathrm{SS}}$ for the passivated samples. It means that the $\tau_{\text {meas }}$ is very close to $\tau_{\mathrm{b}}$ in case of thick samples. Figure 7 shows the detailed laser e-PCD results from three selected samples in different lifetime ranges.
One can observe an increasing lifetime in the first part of the decay curves (at high injection levels) which is related to the decreasing rate of the surface and the Auger recombination. The higher the bulk lifetime and diffusion length, the longer the time period of the surface recombination influence as depicted in Figures $7 \mathrm{~b}$ and 7c. This phenomenon is clearly visible by comparing the lifetime curves from the passivated and as-sliced samples. In the low lifetime range (Fig. 7a) the curves practically do not differ, while in the millisecond lifetime range they become nearly equal only after a period of time comparable to the bulk lifetime or even longer.

In the present industrial practice the carrier lifetime value is reported typically at $10^{15} \mathrm{~cm}^{-3}$. Thus, we examined these $\tau_{\text {meas }}\left(\Delta n=10^{15} \mathrm{~cm}^{-3}\right)$ values in detail (Fig. 7, yellow signs). The lifetime values did not differ more than $20 \%$ from bulk lifetime in any of the samples as we can see in Figure 8 . The excellent correlation between the results 


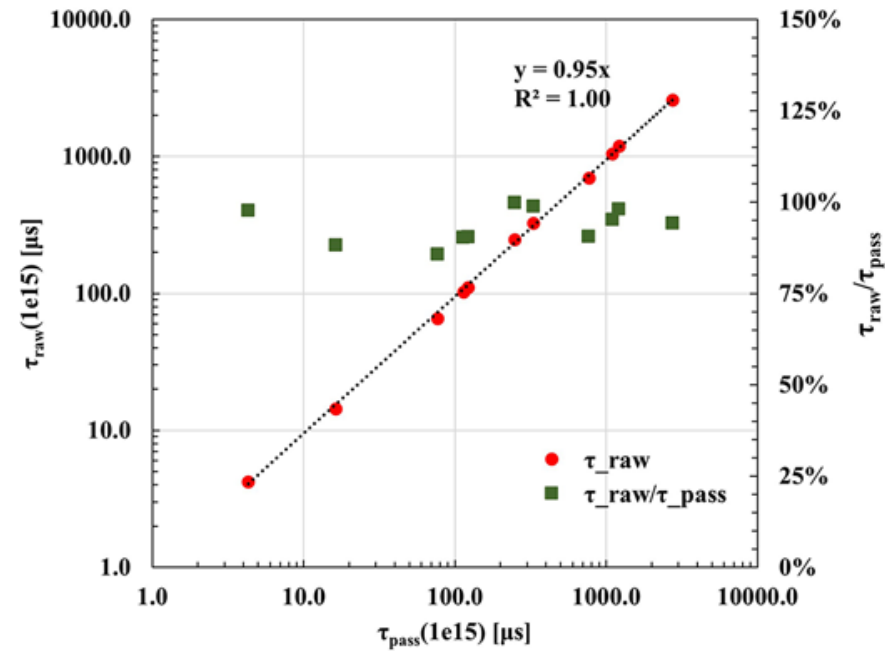

Fig. 8. Excellent correlation found between the lifetime values measured at as-sliced and passivated surfaces at $\Delta n=10^{15} \mathrm{~cm}^{-3}$ injection level in the whole lifetime range of interest. The smallest lifetime sample are compared at $\Delta n=10^{14} \mathrm{~cm}^{-3}$.

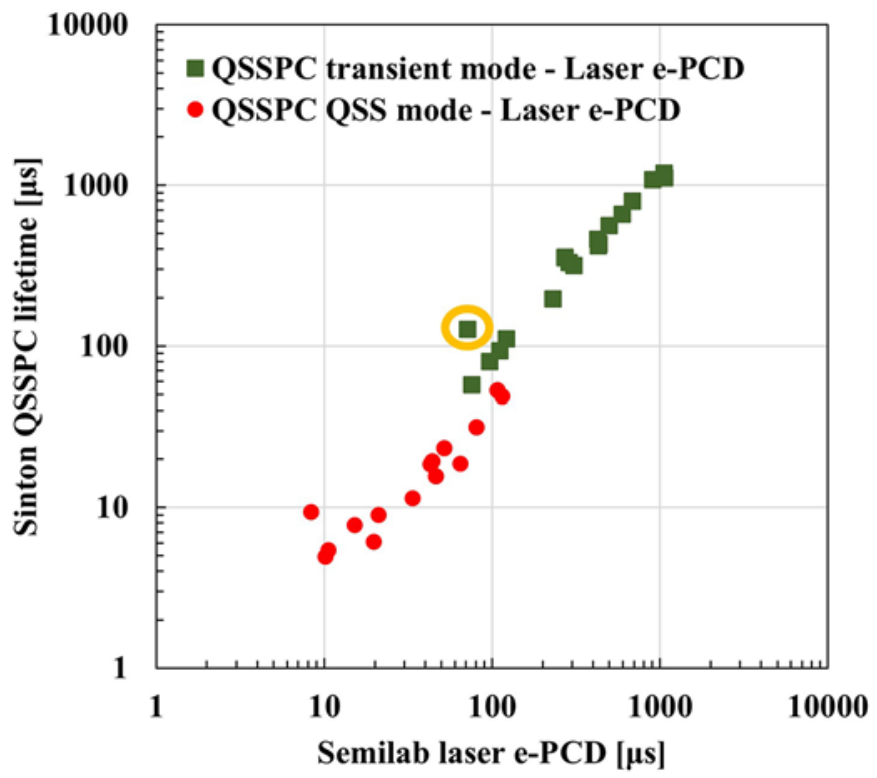

Fig. 9. Comparison of lifetime values obtained using QSSPC and laser e-PCD techniques. Correlation to QSSPC is excellent in its transient mode. The circled out-of-trend value corresponds to a sample featuring especially strong ILD. The large discrepancy between the QSS and transient mode evaluation indicates the importance of the capability of laser e-PCD providing unified transient mode evaluation in the entire lifetime range.

measured on as-sliced and passivated surfaces indicates that the surface quality does not affect $\tau_{\text {meas }}\left(\Delta n=10^{15} \mathrm{~cm}^{-3}\right)$ significantly even in this low $S_{\text {front }}$ range. It further confirms the proper choice of laser wavelength and power.

The comparison of carrier lifetime values reported at $10^{15} \mathrm{~cm}^{-3}$ injection level by the optimized WT-1200IL setup of laser e-PCD $(1064 \mathrm{~nm}$, elevated power $)$ and a
Sinton BCT-400 system using flash lamp is depicted in Figure 9. We measured Cz-mono Ga-doped silicon slabs in a wide lifetime $(10 \mu \mathrm{s}<\tau<1000 \mu \mathrm{s})$ and resistivity range $(0.1 \Omega \mathrm{cm}<\rho<1.3 \Omega \mathrm{cm})$. QSSPC measurements were performed in transient mode first. Long lifetime samples $(\tau>200 \mu \mathrm{s})$ were measured only using transient mode, samples featuring low lifetime $(\tau<50 \mu \mathrm{s})$ were re-measured in QSS mode, and only the QSS mode results are considered for the comparison. In the intermediate lifetime range, measurements were accomplished using both the transient and QSS modes and included in the comparison.

In most cases, the laser e-PCD results agree very well to transient mode of QSSPC results. The outlier (marked with yellow circle) indicates a sample with non-usual injection level dependence, an effect which shall be investigated in detail. However, very large discrepancy is found in the lifetime values between QSS and transient mode of both our tool and the same BCT-400 system in the intermediate lifetime range (might be over a factor of 3 ) originated from the very different evaluation method. It indicates the importance of the unique capability of the laser e-PCD technique providing unified transient mode evaluation in the entire lifetime range naturally avoiding such discrepancy issues.

\section{Conclusions}

We optimized the laser excitation parameters in Semilab's laser e-PCD system. This resulted in enhanced accuracy of the reported lifetime value and in the extended injection level range in which the carrier lifetime can be determined. We developed a 1-D simulation of the measurement process to estimate the accuracy of the results. Both simulation and experimental results showed that the improved laser parameters enables to report near-bulk lifetime values at $\Delta n=10^{15} \mathrm{~cm}^{-3}$ carrier concentration and below from all industrially relevant mono-Si samples. We observed an excellent correlation between e-PCD lifetime results on as-sliced and chemically passivated front surface Si slabs in the entire lifetime range of interest which indicates the independence of the accuracy of the measurement from the surface quality. The comparison with the QSSPC method clearly indicates the important benefit of using laser due to the unified transient mode evaluation. Several simplifications were applied for the evaluation of transients but providing valid results at $\Delta n=10^{15} \mathrm{~cm}^{-3}$ injection level. The complete compensation of the surface recombination effects and the accurate computation of the carrier lifetimes at higher injection levels as well requires to develop more complex transient evaluation routines and PCD simulations which we plan to achieve in the next phase of our laser e-PCD development.

\section{Author contribution statement}

I. Saegh and G. Paráda contributed the tool development, $\mathrm{P}$. Tüttö designed the detector and contributed to the interpretation of the results. X. Dong contributed in the measurement analyses and performed the comparative 
measurements. H. Deng, S. Wang and X. Chen contributed in the measurements and provided the samples. Z. Verdon and Cs. Jobbágy optimized the chemical passivation for Si ingots. M. Kovács supported the understanding of the results with finite-element simulations. D. Krisztián performed the laser e-PCD measurements and analysis, planned and carried out the simulations, drafted the manuscript and designed the figures. F. Korsós were involved in planning, supervised the work and contributed to the final version of the manuscript. All authors discussed the results and commented on the manuscript.

We thank Ferenc Simon for useful discussions. This research was supported by the Ministry of Innovation and Technology and the National Research, Development and Innovation Office (NKFIH) within the Quantum Information National Laboratory. D. Krisztián acknowledge the support of the doctoral student scholarship program of the co-operative doctoral program of the Ministry for Innovation and Technology from the source of the National Research, Development and Innovation Fund.

\section{References}

1. https://itrpv.vdma.org/

2. F. Korsós, L. Roszol, F. Jay, J. Veirman, A.D. Draoua, M. Albaric, T. Szarvas, Z. Kiss, A. Szabó, I. Soczó, Gy. Nádudvari, N. Laurent, Efficiency limiting crystal defects in monocrystalline silicon and their characterization in production, Solar Energy Mater. Solar Cells 186, 217 (2018)

3. D.K. Schroder, Semiconductor Material and Device Characterization (Wiley, New York, 1990)

4. R.A. Sinton, A. Cuevas, Contactless determination of current-voltage characteristics and minority-carrier lifetimes in semiconductors from quasi-steady-state photoconductance, Appl. Phys. Lett. 69, 2510 (1996)

5. T.S. Horányi, T. Pavelka, P. Tüttö, In situ bulk lifetime measurement on silicon with a chemically passivated surface, Appl. Surf. Sci. 63, 306 (1993)

6. S. Rein, T. Rehrl, W. Warta, S.W. Glunz, Lifetime spectroscopy for defect characterization: systematic analysis of the possibilities and restrictions, J. Appl. Phys. 91, 2059 (2002)

7. J. Schmidt, D. Macdonald, Recombination activity of irongallium and iron-indium pairs in silicon, J. Appl. Phys. 97, 113712 (2005)
8. J.D. Murphy, M. Al-Amin, K. Bothe, M. Olmo, V.V. Voronkov, R.J. Falster, The effect of oxide precipitates on minority carrier lifetime in n-type silicon, J. Appl. Phys. 118, 215706 (2015)

9. G. Paráda, F. Korsós, P. Tüttö, Transient Method for Lifetime Characterization of Monocrystalline Si Ingots, in 29th European Photovoltaic Solar Energy Conference and Exhibition (2014), pp. 1264-1266

10. S. Bowden, R.A. Sinton, Determining lifetime in silicon blocks and wafers with accurate expressions for carrier density, J. Appl. Phys. 102, 124501 (2007)

11. J.S. Swirhun, R.A. Sinton, M. Keith Forsyth, T. Mankad, Contactless measurement of minority carrier lifetime in silicon ingots and bricks, Progr. Photovolt. 19, 313 (2011)

12. M. Goodarzi, R. Sinton, D. Macdonald, Improving transient photoconductance lifetime measurements on ingots with deeper photogeneration, AIP Conf. Proc. 1999, 020008 (2018)

13. M. Goodarzi, R. Sinton, D. Macdonald, Quasi-steady-state photoconductance bulk lifetime measurements on silicon ingots with deeper photogeneration, AIP Adv. 9, 015128 (2019)

14. M. Goodarzi, R. Sinton, D. Chung, B. Mitchell, T. Trupke, D. Macdonald, A comparison between quasi-steady state and transient photoconductance lifetimes in silicon ingots: simulations and measurements, in IEEE 44th Photovoltaic Specialist Conference (PVSC) (2017), pp. 2707-2710

15. P. Blood, J.W. Orton, The electrical characterisation of semiconductors, Rep. Prog. Phys. 41, 157 (1978)

16. J. Schmidt, Measurement of differential and actual recombination parameters on crystalline silicon wafers [solar cells, IEEE Trans. Electr. Dev. 46, 2018 (1999)

17. M.A. Green, Self-consistent optical parameters of intrinsic silicon at $300 \mathrm{k}$ including temperature coefficients, Solar Energy Mater. Solar Cells 92, 1305 (2008)

18. A. Richter, F. Werner, A. Cuevas, J. Schmidt, S.W. Glunz, Improved parameterization of auger recombination in silicon, Energy Procedia 27, 88 (2012)

19. F. Dannhauser, Die abhängigkeit der trägerbeweglichkeit in silizium von der konzentration der freien ladungsträger-i, Solid State Electr. 15, 1371 (1972)

20. J. Krausse, Die abhängigkeit der trägerbeweglichkeit in silizium von der konzentration der freien ladungsträger-ii, Solid State Electr. 15, 1377 (1972)

21. D. Macdonald, L.J. Geerligs, Recombination activity of interstitial iron and other transition metal point defects in p- and n-type crystalline silicon, Appl. Phys. Lett. 85, 4061 (2004)

Cite this article as: Dávid Krisztián, Ferenc Korsós, Ilias Saegh, Gábor Paráda, Martin Kovács, Zita Verdon, Csaba Jobbágy, Péter Tüttő, Xueqian Dong, Hao Deng, Shasha Wang, Xiaobo Chen, Improved accuracy of eddy-current sensor based carrier lifetime measurement using laser excitation, EPJ Photovoltaics 13, 3 (2022) 